\title{
Increased Systolic Blood Pressure Driven Skeletal Muscle Activation Following Stroke: A Causality Study
}

\author{
Ajay K Verma ${ }^{1}$, Da Xu ${ }^{2}$, Nandu Goswami ${ }^{3}$, Amanmeet Garg ${ }^{2}$, Franz Fazekas ${ }^{3}$, Andreas Rössler ${ }^{3}$, \\ Andrew Blaber ${ }^{2}$, Reza Fazel-Rezai ${ }^{1}$, Kouhyar Tavakolian ${ }^{1,2}$ \\ ${ }^{1}$ University of North Dakota, Grand Forks, United States \\ ${ }^{2}$ Simon Fraser University, Burnaby, Canada \\ ${ }^{3}$ Medical University of Graz, Graz, Austria
}

\begin{abstract}
Older individuals following stroke have a higher incidence of orthostatic hypotension, syncope and fall risk. Thus, in a pilot study, we investigated the relationship between the arterial and skeletal muscle pump baroreflexes in 8 older (63 \pm 4 years) stroke patients (208 \pm 14 days post-insult) and 8 age-matched healthy controls (62 \pm 7 years) during quiet standing.

Simultaneous continuous non-invasive lower leg muscle activity, electromyography (EMG), 3-lead electrocardiogram (ECG) and blood pressure were recorded continuously during the 5-minute stand portion of a sit-to-stand test. Causality was analyzed between $E M G$ and systolic blood pressure (SBP) in the last 4 minutes of standing. Data were segmented into a time window of 45 seconds, translated with an overlap of 5 seconds on the entire 4-minute data. Convergent cross mapping (CCM) was used for studying the causality between the blood pressure and EMG signals. Spontaneous baroreflex sensitivity (BRS) was calculated using the sequence method.

Between the two groups, there was no difference in spontaneous BRS (stroke: $4.85 \pm 1.52 \mathrm{~ms} / \mathrm{mmHg}$; control: $4.27 \pm 1.68 \mathrm{~ms} / \mathrm{mmHg} ; p=0.48$ ) or causality from $E M G$ towards SBP (stroke: 0.62 \pm 0.15 ; control: $0.67 \pm 0.05$, $p=0.38$ ). The causal relationship from SBP to EMG was greater in stroke patients $(0.48 \pm 0.05)$ compared to controls $(0.41 \pm 0.05, \quad p<0.05)$. Although arterial baroreflex was not different between groups, elevated drive from SBP to EMG activity suggests a potential compensatory action by the baroreflex-mediated muscle pump activation towards blood pressure regulation following stroke.
\end{abstract}

\section{Introduction}

Orthostatic hypotension is often experienced by older persons and in populations with neurodegenerative diseases during standing [1]. Standing causes blood pooling in the lower body extremities due to gravity. During an orthostatic challenge, blood pressure is maintained by a decrease in vagal activity and increase in sympathetic activity resulting in increased heart rate and peripheral vascular resistance. Calf skeletal muscles are known to increase venous return by compressing the underlying veins (skeletal muscle pump) in order to maintain blood pressure while standing [2]. The role of lower leg muscles is, thus, essential for blood pressure regulation, as under low venous return baroreflex mechanism would have limited effect on blood pressure regulation.

Previously, calf skeletal muscles and systolic blood pressure (SBP) have been shown to have linear interaction during quiet standing in order to maintain stable posture [3]. However, the directional information exchange (causality) between EMG and SBP, and any alterations under the pathological conditions, are not clearly understood. Causality is a process of combining cause and its effect. Thus, the causal behavior of systems is critical in quantifying systems functioning. Causal information can be used for monitoring purposes as in causally linked systems cause in one system would be expected to create an effect in another. A decline in directional information transfer between such linked systems could be indicative of system impairment. To avail causality advantages it has been extensively studied in physiology for analyzing the existence of a directional link between systems [4-6].

Patients with a history of stroke are known to have difficulties in maintaining an upright posture [7]. In the current research, we investigated the directional interaction between EMG and SBP in patients with stroke history and age-matched healthy controls to quantify alteration in causality between muscle pump activity and blood pressure (EMG $\leftrightarrow \mathrm{SBP}$ ) as a consequence of stroke. Non-linear convergent cross mapping (CCM) method was 
used for analyzing EMG $\leftrightarrow \mathrm{SBP}$ causality during an orthostatic challenge to investigate the existence of compensatory causal activity following a stroke to facilitate stable upright posture. Choice of CCM was based on its superior performance over Granger Causality on signals that are non-linear and non-stationary in nature inherently [8].

\section{Methods}

\subsection{Data acquisition}

Data was acquired under sit-to-stand protocol from 8 stroke patients (age: $63 \pm 4$ years) and 8 age-matched healthy controls (age: 62 \pm 7 years). Participants sat on a bed, which was adjusted to their knee height. Following 5 minutes of sitting at rest, participants were assisted in quiet standing for the next 5 minutes on a force plate, while looking straight ahead and with their feet shoulder width apart. Participants were instructed to breathe normally and sway or shift their weight if they felt uncomfortable, but asked to stay as relaxed and still as possible. After 5 minutes of quiet standing, participants were assisted to resume the initial seated position for another 3 minutes.

All protocols were performed between 7-11 AM in a quiet room maintained at approximately $25^{\circ} \mathrm{C}$. Participants were asked to refrain from coffee or other stimulants 24 hours prior to experimentation. The project received approval from the Ethics Committee of the Medical University of Graz.

Arterial blood pressure was measured with noninvasive finger photoplethysmography (Finometer, Finapres Medical Systems B.V., Amsterdam, The Netherlands), heart rate was assessed using bipolar 3 lead ECG (FD-13, Fukuda Denshi Co. Ltd, Tokyo, Japan), center of pressure in medio-lateral (COPx) and anteriorposterior (COPy) direction was obtained using force plate (AccuSway, Advanced Mechanical Technology, Inc., Watertown, MA, USA), and EMG of bilateral gastrocnemius muscles (MyoSystem 1200, Noraxon U.S.A Inc., Scottsdale, AZ, USA) were obtained during the entire sit-to-stand protocol.

\subsection{Convergent cross mapping}

CCM is a non-linear approach to infer causality between bivariate time series [9, 10]. Causality between the variables is determined by studying the correspondence between non-linear state space; reconstructed using lagged coordinates of two variables (shadow manifolds). For two time series $\mathrm{X}$ and $\mathrm{Y}$, the causal information flow from $\mathrm{X}$ to $\mathrm{Y}(\mathrm{X} \rightarrow \mathrm{Y})$ is determined by quantifying the correspondence between original $\mathrm{X}$ and $\mathrm{X}$ estimated $(\widehat{\mathrm{X}})$, using manifold of $\mathrm{Y}$
$\left(M_{Y}\right)$ using the Pearson correlation coefficient $(\rho)$. Thus, if there is causal information flowing from $\mathrm{X}$ to $\mathrm{Y}$, the manifold of $\mathrm{Y}$ would be helpful in estimating $\mathrm{X}$ while the manifold of $X$ would contain no information helpful in estimating states of $\mathrm{Y}$. Higher the correlation between original and estimated states stronger would be the causal information flow. This notion of inferring causality is contrary to the one proposed by Granger where the driver is used to predict response, hence it is termed cross mapping [11]. Mathematically, causal information from $\mathrm{X}$ to $\mathrm{Y}$ can be represented as $\mathrm{X} \rightarrow \mathrm{Y}=\rho\left(\mathrm{X}, \widehat{\mathrm{X}} \mid \mathrm{M}_{\mathrm{Y}}\right.$, similarly, the equation for causal information flowing from $Y$ to $X$ can be written as $\mathrm{Y} \rightarrow \mathrm{X}=\rho\left(\mathrm{Y}, \widehat{\mathrm{Y}} \mid \mathrm{M}_{\mathrm{X}}\right)$. The convergence of this correlation increases with the increase in data length, thus, CCM separates causation from correlation [9].

Using CCM method unidirectional $(\mathrm{X} \rightarrow \mathrm{Y}$ or $\mathrm{Y} \rightarrow \mathrm{X})$ or bi-directional $(\mathrm{X} \leftrightarrow \mathrm{Y}$ ) causality can be determined. Under the ideal case of unidirectional $(\mathrm{X} \rightarrow \mathrm{Y})$ causality $0<X \rightarrow Y \leq 1$ while $Y \rightarrow X=0$. In the case of bidirectional causality, both $\mathrm{X}$ and $\mathrm{Y}$ can be estimated using a manifold of $\mathrm{Y}$ and $\mathrm{X}$, respectively. Under such condition, $0<\mathrm{X} \rightarrow \mathrm{Y} \leq 1$ and $0<\mathrm{Y} \rightarrow \mathrm{X} \leq 1$, the stronger causal behavior can be determined by calculating the difference of two causal events. If $\mathrm{X} \rightarrow \mathrm{Y}$ is stronger than $\mathrm{Y} \rightarrow \mathrm{X}$ then, $\rho\left(X, \widehat{X} \mid M_{Y}\right) \rho\left(Y, \widehat{Y} \mid M_{X}\right)$ would be greater than zero, else it would be negative.

The detailed algorithmic explanation of CCM is presented in [12]. In literature CCM has been successful in revealing the existence of causality in non-linear physiological signals $[8,11,13]$; its performance has also been superior in comparison to linear Granger causality when applied on non-linear physiological signals [8]. With its advantage, it is expected to unearth complex directional interaction between SBP and EMG.

\subsection{Data processing}

Last four minutes of standing data acquired was considered for causality analysis. A QRS complex of ECG signal was detected using a Pan Tompkins algorithm [14], after this, a sequence of R-R interval was obtained as a time difference between two adjacent QRS complexes. Beat-by-beat SBP time series was obtained as maximum pressure values of the blood pressure waveform within each R-R interval. A moving average filter with a cut-off frequency of $5 \mathrm{~Hz}$ was used to obtain EMG envelope. From EMG envelope beat-by-beat EMG time series was obtained as a mean rectified value within each heartbeat. Beat-by-beat SBP and EMG time series were interpolated using spline interpolation to generate a series of evenly spaced samples at a sampling rate of 100 $\mathrm{Hz}$ before causality analysis. To perform CCM analysis embedding dimension $(E=4)$ was chosen using false nearest neighbor [15] and the delay was chosen to be 2 samples. 
The data was segmented into short time segments of 45 seconds, this window was then translated with an overlap of 5 seconds over a 4-minute period, and this step resulted into EMG-SBP causality being computed into 5 time windows. In each window, the correlation coefficient value at which $\mathrm{EMG} \rightarrow \mathrm{SBP}$ and $\mathrm{SBP} \rightarrow \mathrm{EMG}$ converged was taken into account. Per subject causal behavior $(\mathrm{EMG} \rightarrow \mathrm{SBP}$ or $\mathrm{SBP} \rightarrow \mathrm{EMG})$ was determined by averaging these causality values over 5 segments. The significance level for the correlation was set at 0.05 .

Arterial baroreflex sensitivity (BRS) was calculated using the sequence method [16]. An algorithm for BRS calculation required R-R (ms), SBP $(\mathrm{mmHg})$, and a threshold for absolute inter-beat change of SBP as an input parameter. A sequence of three or more beats for which R-R and SBP values increased or decreased in the same direction with an absolute change in SBP greater than $0.5 \mathrm{~mm} \mathrm{Hg}$ were considered.

\section{Results}

Non-significant difference $(\mathrm{p}=0.48)$ in BRS was found between the control $(4.27 \pm 1.68 \mathrm{~ms} / \mathrm{mmHg})$ and stroke $(4.85 \pm 1.52 \mathrm{~ms} / \mathrm{mmHg})$ groups. The false nearest neighbor was used to choose the optimal dimension of reconstruction. False nearest neighbor achieved its minimum on an average at an embedding dimension value of 4 and higher. Thus, 4 dimensions of reconstruction were used for CCM causality analysis. Causality analysis results were suggestive of the existence of bidirectional causality between EMG and SBP in both groups; as the causal activity of both EMG $\rightarrow$ SBP and SBP $\rightarrow$ EMG was observed (Table 1). Skeletal muscle pump driven SBP control (EMG $\rightarrow$ SBP) was found to be significantly higher in both groups (stroke: $0.62 \pm 0.15$; control: $0.67 \pm 0.05)$ compared to reverse causality of SBP $\rightarrow$ EMG (stroke: $0.48 \pm 0.05$; control: $0.41 \pm 0.05$ ). A non-significant difference in the $\mathrm{EMG} \rightarrow \mathrm{SBP}$ causality $(p=0.38)$ in the two groups was found while in stroke group $\mathrm{SBP} \rightarrow$ EMG causality value was significantly higher $(\mathrm{p}<0.05)$ than the control group (Figure 1$)$.

Table 1. Average EMG $\leftrightarrow$ SBP causality for two groups $(\mathrm{N}=8)$. * represents significantly different causal strength in comparison to reverse causal strength.

\begin{tabular}{|c|c|c|}
\hline Signal/Group & Control & Stroke \\
\hline $\mathrm{EMG} \rightarrow \mathrm{SBP}$ & $0.67 \pm 0.05$ & $0.62 \pm 0.15$ \\
\hline $\mathrm{SBP} \rightarrow \mathrm{EMG}$ & $0.41 \pm 0.05^{*}$ & $0.48 \pm 0.05^{*}$ \\
\hline
\end{tabular}

\section{Discussions}

The current pilot study to the best of author's knowledge is the first study to apply causality based approach to quantify mechanisms involved in blood pressure regulation under orthostatic challenge following a stroke. We investigated arterial baroreflex and skeletal muscle pump driven regulation of systolic blood pressure in healthy older participants and age-matched patients with a history of stroke under orthostatic stress induced in the form of sit-to-stand test. Baroreflex sensitivity measured using the sequence method showed nonsignificant $(\mathrm{p}=0.48)$ change in stroke patients $(4.85 \pm 1.52$ $\mathrm{ms} / \mathrm{mmHg})$ in comparison to controls $(4.27 \pm 1.68$ $\mathrm{ms} / \mathrm{mmHg}$ ) suggesting unchanged or fast recovered arterial baroreflex mechanism of blood pressure regulation following stroke.

Both groups showed bidirectional causal interaction between EMG and SBP (Table 1). Skeletal muscle pump driven SBP control $(\mathrm{EMG} \rightarrow \mathrm{SBP})$ was significantly higher $(p<0.01)$ than reverse causality of $\mathrm{SBP} \rightarrow$ EMG in both groups (Table 1), thus highlighting an essential role of skeletal muscles in blood pressure regulation. A non significant $(\mathrm{p}=0.38)$ change was noticed in the strength of muscle pump driven SBP control in the stroke group compared to controls indicating that mechanical muscle pump functionality was not likely affected by stroke.

In stroke group, significantly greater $(p<0.05)$ $\mathrm{SBP} \rightarrow \mathrm{EMG}$ causal activity was observed, which is suggestive of stronger SBP driven alterations in skeletal muscle activation after stroke.

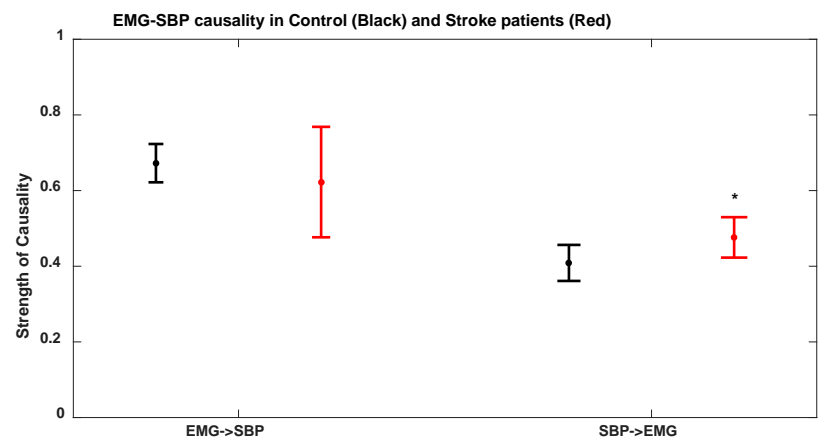

Figure 1 . Average $\mathrm{EMG} \rightarrow \mathrm{SBP}$ and $\mathrm{SBP} \rightarrow \mathrm{EMG}$ causality values for controls and stroke patients. * represents stroke patients with a significantly different mean in comparison to mean of the control population.

Significant change in $\mathrm{SBP} \rightarrow \mathrm{EMG}$ causality, accompanied by a non-significant change in BRS, indicate reliance of skeletal muscles on SBP driven changes in order to regulate blood pressure. Thus, baroreflex-mediated muscle pump appears to play a compensatory role following a stroke in order to regulate blood pressure.

In the current study, alterations in cardiovascular and, postural systems interaction following stroke was quantified using representative signals of SBP and EMG, 
respectively. The current study did not analyze center of pressure (COP) signal, increased postural sway following stroke has been reported in the literature [17]. Previous studies have shown that an interaction between cardiovascular and postural systems marked by EMG, $\mathrm{SBP}$, and $\mathrm{COPr}$ is an essential mechanism to maintain stable upright posture under orthostatic challenge. Furthermore, prior preliminary works have also shown the existence of feedback causality in between cardiopostural variables [18-20]. Thus, performing causality analysis of EMG and SBP with COP signal will further strengthen our understanding of causal cardio-postural interaction following stroke.

\section{Conclusions and future work}

Outcomes of current pilot research lead us to conclude three major findings: 1) skeletal muscle pump plays a pivotal role in blood pressure regulation as evident from significantly stronger $(p<0.01) \quad \mathrm{EMG} \rightarrow \mathrm{SBP}$ activity in controls as well as in stroke patients in comparison to reverse causality of $\mathrm{SBP} \rightarrow \mathrm{EMG}$; 2) baroreflex sensitivity does not carry residual effects of stroke as discernable by non-significant $(\mathrm{P}=0.48)$ change in BRS between stroke and control groups; and 3) SBP $\rightarrow$ EMG activity plays a compensatory role in the regulation of blood pressure. Despite no significant changes in BRS, stroke patients exhibited markedly greater $\mathrm{SBP} \rightarrow \mathrm{EMG}$ causality than the controls, thus highlighting blood pressure regulation mechanism through baroreflex-mediated muscle pump.

Future work shall include COP signal while performing causality analysis in order to complete cardiopostural control loop for a better understanding of physiological balance mechanism activity following stroke. To generalize the finding of the current study, a similar data analysis on bigger cohort would be required.

\section{References}

[1] Gauer RL. Evaluation of Syncope. American family physician. 2011;84(6):640.

[2] Masterson M, Morgan A. The Role of Lower Leg Muscle Activity in Blood Pressure Maintenance of Older Adults. Clin Kinesiol. 2006;60(16):8-18.

[3] Garg A, Xu D, Laurin A, Blaber AP. Physiological interdependence of the cardiovascular and postural control systems under orthostatic stress. Am J Physiol Heart Circ Physiol. 2014;307(2):H259-64.

[4] Porta A, Faes L. Assessing causality in brain dynamics and cardiovascular control. Philosophical Transactions of the Royal Society of London A: Mathematical, Physical and Engineering Sciences.2013;371(1997):20120517.

[5] Bressler SL, Seth AK. Wiener-Granger Causality: A well established methodology. Neuroimage. 2011;58(2):323-9.

[6] Porta A, Faes L. Wiener - Granger Causality in Network Physiology With Applications to Cardiovascular Control and Neuroscience. Proc IEEE. 2016;104(2):282-309.
[7] Langhorne P, Coupar F, Pollock A. Motor recovery after stroke: a systematic review. Lancet Neurol. 2009;8(8):741-54.

[8] Schiecke K, Pester B, Piper D, Benninger F, Feucht M, Witte $\mathrm{H}$. Nonlinear Directed Interactions between Heart Rate Variability and EEG Activity in Children with Temporal Lobe Epilepsy. IEEE Trans Biomed Eng. 2016;9294(c).

[9] Sugihara G, May R, Ye H, Hsieh C, Deyle E, Fogarty M, Munch S. Detecting Causality in Complex Ecosystems. 2012;338(6106):496-500.

[10] Krakovská A, Jakubík J, Budáčová H, Holecyová M. Causality studied in reconstructed state space. Examples of uni-directionally connected chaotic systems. arXiv Prepr arXiv151100505 2015 Nov 2. 2015:1-41.

[11] Schiecke K, Pester B, Feucht M, Leistritz L, Witte H. Convergent Cross Mapping: Basic concept, influence of estimation parameters and practical application. IEEE EMBC. 2015:7418-21.

[12] Sugihara G, May R, Ye H, Hsieh C, Deyle E, Fogarty M. Supplementary Materials for Detecting Causality in Complex Ecosystems. 2012;

[13] Heskamp L, Meel-van den Abeelen AS, Lagro J, Claassen JA. Convergent cross mapping : a promising technique for cerebral autoregulation estimation. IJCNMH. 2014; 1:S20.

[14] Pan J, Willis J. A Real-Time QRS Detection Algorithm. 1985;1(3):230-6.

[15] Kennel M, Brown R. Determining embedding dimension for phase-space reconstruction using geometrical construction. Phys Rev A. 1992;45(6); 3403.

[16] Carrington CA, White MJ. Spontaneous baroreflex sensitivity in young and older people during voluntary and electrically evoked isometric exercise. Age Ageing. 2002;31(5):359-64.

[17] Genthon N, Rougier P, Gissot AS, Froger J, Pelissier J, Perennou D. Contribution of Each Lower Limb to Upright Standing in Stroke Patients. Stroke. 2008;39(6):1793-9.

[18] Verma AK, Garg A, Blaber A, Fazel-Rezai R Tavakolian K. Causality in the Cardio-Postural Interactions during Quiet Stance. Computing in Cardiology. 2015:373-6.

[19] Verma AK, Garg A, Blaber A, Fazel-Rezai R, Tavakolian K. Causal Cardio-Postural Interaction Under Orthostatic Stress. J Med Device. 2016;10(June):6-7.

[20] Verma AK, Garg A, Blaber A, Fazel-Rezai R, Tavakolian K. Causality Detection in Cardio-Postural Interaction under Orthostatic Stress Induced by Quiet Standing using Transfer Entropy. IEEE EIT. 2016:633-7.

Address for correspondence

Kouhyar Tavakolian

Department of Electrical Engineering

243 Centennial Drive Stop 7165

Grand Forks, ND 58202

Kouhyar@und.edu 